\title{
Modulation of high-frequency quasi-periodic oscillations by relativistic effects
}

\author{
Michal Bursa \\ Astronomical Institute, Academy of Sciences, Boční II, CZ-14131 Prague, Czech Republic \\ email: bursa@astro.cas.cz
}

\begin{abstract}
Two distinct frequencies are often present in frequency power spectra of low-mass $\mathrm{X}$-ray binary lightcurves. These frequencies are closely related to each other and there is a strong evidence that they have origin in the orbital motion in an accretion disc. A mechanism has to be in act that provides a modulation of emergent flux at observed frequencies - not necessarily the actual frequencies at which the fluid oscillates. Using numerical ray-tracing we show that strong gravity has an effect on light modulation and may itself be responsible for significant part of variations in observed lightcurves.
\end{abstract}

Keywords. Accretion disks - gravitational lensing - X-rays: binaries

\section{Introduction}

Observations show that the solely presence of a thin accretion disc is not sufficient to produce the high-frequency quasi-periodic oscillations (HFQPO), because they are exclusively connected to the spectral state, where the energy spectrum is dominated by a steep power law with some weak thermal disc component. A model is more appropriate, where an outer cool disc is continuously transitioned into or sandwiched by a hot, thick, but optically thin flow (Esin et al. 1998). An optically thin advection-dominated accretion flow is mostly transparent for photons, and therefore general relativistic light bending and lensing effects may gain a particular importance. Significant temporal variations in the observed flux can then be accomplished by global oscillations of such geometrically thick flow, fluid tori.

In order to explore, whether it is possible to obtain some flux modulation just by effects of strong gravity, we set up a model of a possible accretion configuration, largely simplified to a hot and optically thin luminous torus, optionally surrounded by a cool opaque disc. The torus is considered in a slender approximation, i.e. with its cross-section being much smaller than its distance from the gravity centre (Bursa 2006).

\section{Slender torus model}

We impose on the torus rigid and axisymmetric sinusoidal oscillations in the vertical direction, i.e. parallel to its axis, as well as in the perpendicular radial direction. Such assumption will serve us to model the possible basic global modes found by (Abramowicz et al. 2006). The torus is rigidly displaced from its equilibrium, so that the position of the central circle of maximal pressure varies in time periodically, $r(t)=r_{0}+\delta r \sin \left(\omega_{r} t\right)$, $z(t)=\delta z \sin \left(\omega_{z} t\right)$, with the frequencies of epicyclic motion at a given radius.

Two modes are assumed in numerical calculations described hereby: an incompressible mode and a compressible mode. In the incompressible mode, the equipotential structure and all thermodynamical quantities describing the torus do not vary in time as the torus moves. The compressible mode allows for the redistribution of gas in the torus in a response to changes in the radial distance of the torus centre. 

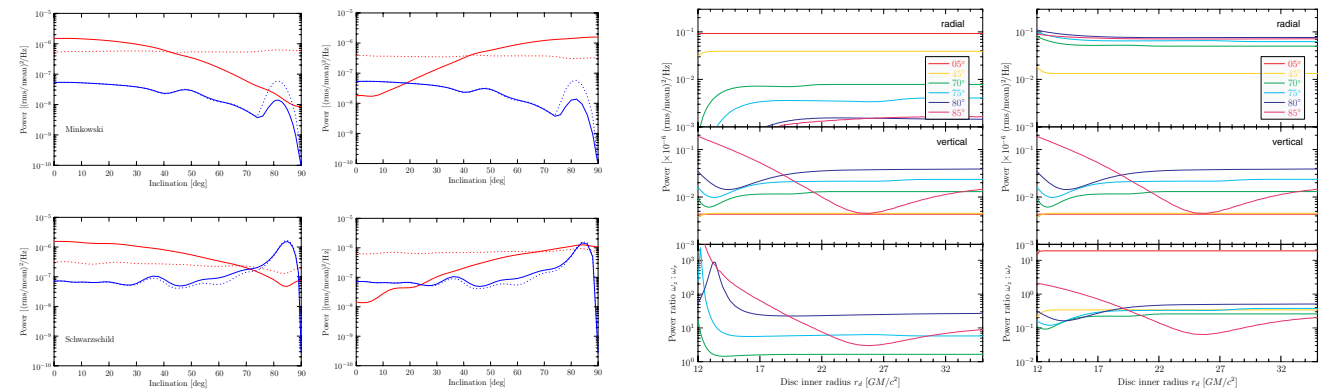

Figure 1. Left pair of panels: the effect of changing observer inclination on the distribution of powers in the radial (red) and vertical (blue) oscillations considering Minkowski and Schwarzschild geometry and the effect of g-factor (solid/dashed lines). The left-most panel shows the compressible mode, the right panel shows the incompressible mode. Right pair of panels: the case of an opaque disc as a function of the torus distance from the gravity centre. Different viewing angles are plotted. The disc lies in the the equatorial plane and goes from infinity down to some terminal radius $r_{d}$, which is a parameter of the model. The disc can extend as close as to the torus, but does not penetrate into it. Left panel shows the compressible mode, the right-most panel shows the incompressible mode. For details, see Bursa (2006).

\section{Inclination and an opaque-disc effect}

Figure 1 compares the dependence of the radial and vertical oscillation powers on changing inclination if the torus is incompressible (left) or compressible (right). We can see that the power in the vertical oscillation stays unchanged, while the radial power is largely affected, particularly if the inclination is changed. There is a clear difference between the red curve progression in the left and right panel. It is caused by the inversion of the luminosity dependence on the torus displacement, which in combination with the effect of g-factor results in a reverse trend of the $\omega_{r}$ power.

There can likely be an outer cool disc surrounding the torus, from which the torus is formed, and which can have a substantial effect on light modulation. The ShakuraSunyaev disc is optically thick and blocks photons crossing the equatorial plane beyond its terminal radius. Most of the stopped photons have been strongly bent and they carry information predominantly about the vertical mode; thus, the presence or lack of an opaque disc may be important for the power distribution in QPO modes, namely the vertical one. Figure demonstrates how the power of the oscillation modes is changed if an opaque disc is present. The presence of a thin disc is important if it does not end far from the torus, but rather within a distance of $\sim 5$ gravitational radii from it, and when the viewing angle is moderate to high. Under these conditions the effect of the torus obscuration by an optically thick medium is capable to substantially change powers in the oscillations, and in particular in the vertical mode.

\section{Acknowledgements}

The author is affiliated with Centre for Theoretical Astrophysics in Prague and his work is supported by the Czech Science Foundation (ref. 205/07/0052) and the Academy of Sciences (ref. KJB300030703).

\section{References}

Abramowicz, M. A., Blaes, O. M., Horák J., Kluźniak, W. \& Rebusco, P. 2006, Classical and Quantum Gravity, 23, 1689

Bursa, M. 2006, High-frequency quasi-periodic oscillations and their modulation by relativistic effects, PhD Thesis (Charles University, Prague)

Esin, A. A., Narayan, R., Cui, W., Grove, J. E. \& Zhang, S.-N. 1998, ApJ, 505, 854 